\title{
Experiment on Bifurcation and Chaos in Coupled Anisochronous Self-Excited Systems: Case of Two Coupled van der Pol-Duffing Oscillators
}

\author{
J. Kengne, ${ }^{1,2}$ F. Kenmogne, ${ }^{3}$ and V. Kamdoum Tamba ${ }^{1,4}$ \\ ${ }^{1}$ Laboratory of Automation and Applied Computer (LAIA), Department of Electrical Engineering, IUT-FV Bandjoun, \\ University of Dschang, P.O. Box 134, Bandjoun, Cameroon \\ ${ }^{2}$ Institute for Smart-Systems Technologies, University of Klagenfurt, Universitätsstraße 65, 9020 Klagenfurt, Austria \\ ${ }^{3}$ Department of Physics, University of Yaoundé 1, P.O. Box 8390, Yaoundé, Cameroon \\ ${ }^{4}$ Laboratory of Electronics, Department of Physics, University of Dschang, P.O. Box 134, Bandjoun, Cameroon
}

Correspondence should be addressed to J. Kengne; kengnemozart@yahoo.fr

Received 29 June 2014; Revised 17 September 2014; Accepted 19 September 2014; Published 29 October 2014

Academic Editor: Mohamed Belhaq

Copyright (c) 2014 J. Kengne et al. This is an open access article distributed under the Creative Commons Attribution License, which permits unrestricted use, distribution, and reproduction in any medium, provided the original work is properly cited.

The analog circuit implementation and the experimental bifurcation analysis of coupled anisochronous self-driven systems modelled by two mutually coupled van der Pol-Duffing oscillators are considered. The coupling between the two oscillators is set in a symmetrical way that linearly depends on the difference of their velocities (i.e., dissipative coupling). Interest in this problem does not decrease because of its significance and possible application in the analysis of different, biological, chemical, and electrical systems (e.g., coupled van der Pol-Duffing electrical system). Regions of quenching behavior as well as conditions for the appearance of Hopf bifurcations are analytically defined. The scenarios/routes to chaos are studied with particular emphasis on the effects of cubic nonlinearity (that is responsible for anisochronism of small oscillations). When monitoring the control parameter, various striking dynamic behaviors are found including period-doubling, symmetry-breaking, multistability, and chaos. An appropriate electronic circuit describing the coupled oscillator is designed and used for the investigations. Experimental results that are consistent with results from theoretical analyses are presented and convincingly show quenching phenomenon as well as bifurcation and chaos.

\section{Introduction}

In recent years, considerable research efforts had been devoted to the analysis of coupled limit-cycle oscillators models due to the useful insight they provide into the collective dynamics of various physical, biological, chemical, and electrical (e.g., coupled van der Pol-Duffing electrical circuit) systems [1-5]. The system of two coupled van der Pol oscillators is one of the canonical models exhibiting the mutual synchronisation behaviour [6]. Coupled selfoscillators demonstrate some classical effects such as phase locking of the oscillations with different ratios of the frequencies [7]. Amplitude death or quenching is possible in case of dissipative coupling $[8,9]$. This phenomenon has been observed experimentally in the system of coupled electromechanical oscillators [10], coupled electronic systems [11], and coupled biological oscillators [11] just to name a few. Multistability, chaos, and nonisochronism are some special effects of the synchronization picture presented by researchers [12-17].

Introducing additional nonlinearity of Duffing type in the original van der Pol equation leads to the so-called van der Pol-Duffing equation for which potential contains a component in the form of the coordinate raised to the fourth power [18-20]. This component models an interesting physical effect, namely, an opportunity of anisochronous oscillations, defined as the dependence of oscillation frequency on oscillation amplitude. Using the method of dynamic regime 
chart, the specific properties of synchronization for coupled autooscillating systems characterized by nonquadratic law of potential dependence on the coordinate were discussed in [18]. For the special case of mutually coupled van der Pol-Duffing oscillators, the structure of the parameter plane (frequency mismatch-coupling strength) was presented. The features of attractors in phase space were examined as well as the arrangement of synchronization tongues and corresponding internal structure in the parameter space. Regions of amplitude death behaviour were also depicted. However the important role of the anisochronism parameter/coefficient (i.e., the cubic nonlinearity parameter of the model) was not sufficiently highlighted. Furthermore, no tractable analytical formulae were provided to define regions of the parameters space corresponding to specific type of behaviours (e.g., Hopf bifurcation, amplitude death, and so on). Also, in order to show the reader the validity of the numerically observed phenomena related to oscillation quenching and bifurcation to chaos of two coupled van der Pol-Duffing oscillators, fullscale experiment with electronic circuits [6] would have been welcomed. With the motivation of shedding more light on the dynamics of such a coupled system, our objective in this paper is threefold: (i) to consider the dynamics of the coupled oscillators and define (analytically) regions of parameters space corresponding to the quenching mode of oscillations; (ii) to investigate the bifurcation structures of the system with particular attention on the effects of anisochronism; (iii) to carry out an experimental study of the system to validate and complement the theoretical results.

The paper is structured as follows. Section 2 describes the mathematical model of the system under investigation and underlines possible symmetries. The coupled van der Pol-Duffing electrical circuit is presented as a prototypal anisochronous self-excited system. In Section 3, conditions for oscillation death behaviour are derived as well as those related to the occurrence of Hopf bifurcations. Section 4 investigates the bifurcation structures of the system numerically with particular emphasis on the effects of cubic nonlinearity of the model. In Section 5, an appropriate analog computer is proposed for the analysis of the dynamic behaviour of the coupled system. Correspondences are established between the coefficients of the system model and the components of the analog simulator. Experimental and numerical results are compared. Some concluding remarks are presented in Section 6.

\section{System Model}

2.1. Description and Analysis of the Model. The dynamics of a system consisting of two mutually coupled van der PolDuffing oscillators considered in this work is described by the following set of equations:

$$
\begin{gathered}
\ddot{x}-\left(\varepsilon-x^{2}\right) \dot{x}+x+\beta x^{3}-\mu(\dot{y}-\dot{x})=0, \\
\ddot{y}-\left(\varepsilon-y^{2}\right) \dot{y}+(1+\Delta) y+\beta y^{3}-\mu(\dot{x}-\dot{y})=0,
\end{gathered}
$$

where $\Delta$ is the frequency mismatch between the second and the first oscillators and $\varepsilon$ characterizes the excess above the threshold of Andronov-Hopf bifurcation in autonomous oscillators [18], while $\mu$ represents the coupling strength. The parameter $\beta(\beta \geq 0)$ defines the perturbation quantity of the quadratic (single-well) potential and consequently is responsible for anisochronism of small oscillations. Setting $\dot{x}=u$ and $\dot{y}=v$, system (1a) and (1b) can be rewritten as a set of four first-order differential equations in the form

$$
\begin{aligned}
& \dot{x}=u, \\
& \dot{u}=\left(\varepsilon-x^{2}\right) u-x-\beta x^{3}+\mu(v-u), \\
& \dot{y}=v, \\
& \dot{v}=\left(\varepsilon-y^{2}\right) v-(1+\Delta) y-\beta y^{3}+\mu(u-v) .
\end{aligned}
$$

For $\mu=0$, both oscillators are uncoupled and each exhibits a limit cycle attractor. Obviously the coupling employed is a linear feedback which can be viewed as the perturbation of each oscillator by a signal proportional to the difference of their velocities (i.e., dissipative coupling). We note that system $(2 \mathrm{a})-(2 \mathrm{~d})$ is invariant under the transformation:

$$
(x(t), u(t), y(t), v(t)) \Longleftrightarrow(-x(t),-u(t),-y(t),-v(t)) .
$$

Therefore, if $(x(t), u(t), y(t), v(t))$ is a solution of system (2a)-(2d) for a specific set of parameters $\varepsilon, \mu, \Delta$, and $\beta$, then $(-x(t),-u(t),-y(t),-v(t))$ is also a solution for the same parameters set. The fixed point $O(0,0,0,0)$ is a trivial symmetric solution. Consequently, attractors in state space have to be symmetric with respect to the origin; otherwise they must appear in pairs, to restore the exact symmetry of the model equations. This exact symmetry may serve to explain the existence of several attractors in state space.

\subsection{Example of Coupled Anisochronous Self-Excited System.} As a prototypal dynamic system (related to the model defined in (1a) and (1b)), we consider the coupled van der Pol-Duffing electrical circuit depicted in Figure 1. The coupled system consists of two oscillators $X$ (Figure 1(a)) and $Y$ (Figure 1(b)) interconnected via a coupling module (Figure $1(\mathrm{c})$ ). The set of diodes $D_{j}(j=1-8)$ with related op. amplifier is the physical implementation of the nonlinear element/resistor responsible for the complex behavior observed in the coupled system. The current-voltage $(I-V)$ characteristic of this nonlinear resistor [5] can be approximated by a cubic polynomial $I(V)=$ $-a V+b V^{3}(a, b>0)$. The coupling module involves a differential voltage amplifier followed by an inverter stage. In each oscillator stage, a current to voltage converter is series inserted with the capacitor to obtain the input signal for the coupling module. Denoting by $V_{1}$ and $V_{2}$ the voltage across capacitor $C$ involved in the Van der Pol-Duffing oscillators $X$ and $Y$, respectively, the application of Kirchhoff current and voltage laws to the schematic diagram of Figure 1 leads to 


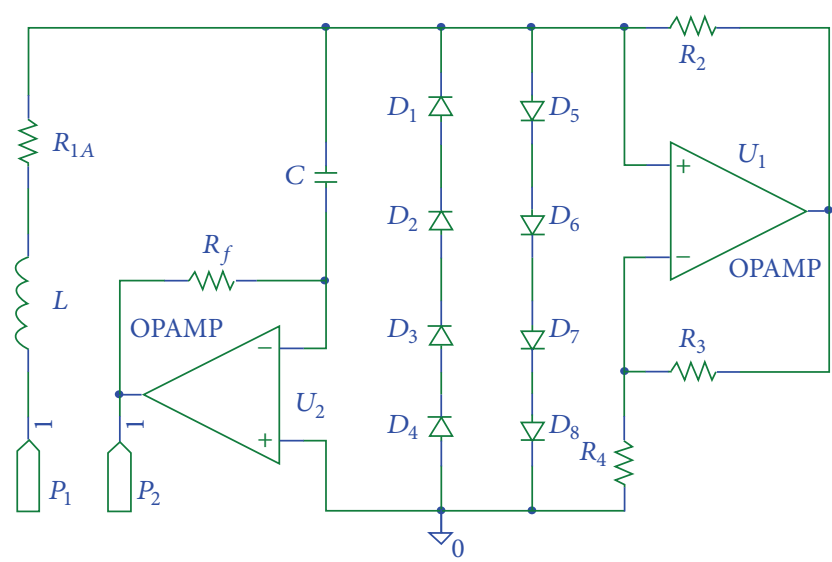

(a)

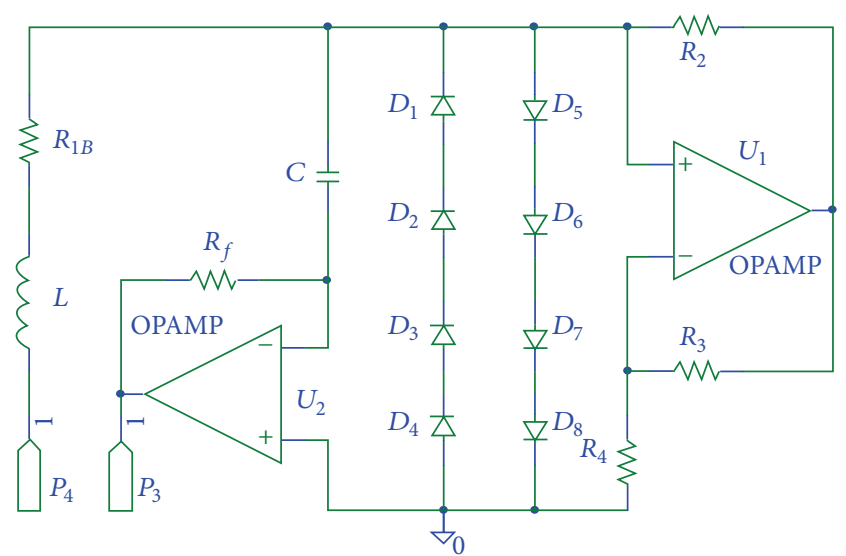

(b)

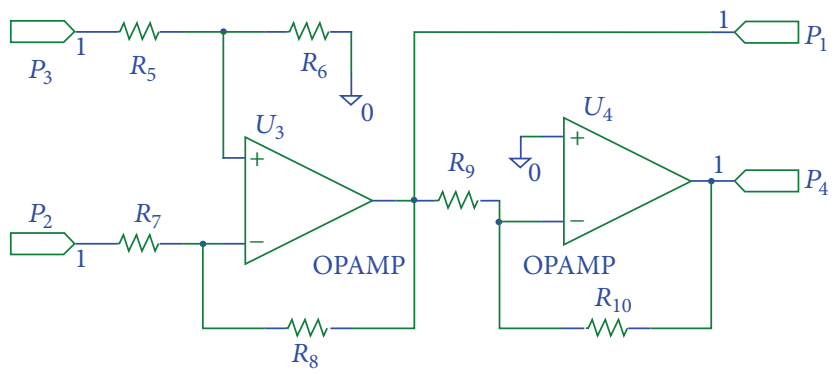

(c)

Figure 1: Scheme of a pair of coupled van der Pol-Duffing electrical system: (a) oscillator X, (b) oscillator $Y$, and (c) coupling module. The relationships between the coupling module's components are $R_{9}=R_{10}, R_{5}=R_{7}, R_{6}=R_{8}$; thus we have $A_{d}=R_{8} / R_{7}$.

the derivation of the following differential equations describing the coupled system:

$$
\begin{aligned}
& L C \frac{d^{2} V_{1}}{d \tau^{2}}+\left[R_{1 A} C+L\left(-a+3 b V_{1}^{2}\right)\right] \frac{d V_{1}}{d \tau}+\left(-a R_{1 A}+1\right) V_{1} \\
& \quad+R_{1} b V_{1}^{3}+A_{d} R_{f} C\left(\frac{d V_{1}}{d \tau}-\frac{d V_{2}}{d \tau}\right)=0, \\
& L C \frac{d^{2} V_{2}}{d \tau^{2}}+\left[R_{1 B} C+L\left(-a+3 b V_{2}^{2}\right)\right] \frac{d V_{2}}{d \tau}+\left(-a R_{1 B}+1\right) V_{2} \\
& \quad+R_{1 B} b V_{2}^{3}+A_{d} R_{f} C\left(\frac{d V_{2}}{d \tau}-\frac{d V_{1}}{d \tau}\right)=0,
\end{aligned}
$$

where $A_{d}$ represents the gain of the differential amplifier stage (see Figure 1 caption). With the following change of variables and parameters:

$$
\begin{gathered}
\tau=t \sqrt{L C}, \quad \rho=\sqrt{\frac{L}{C}}, \quad \mu=A_{d} \frac{R_{f}}{\rho}, \\
V_{\text {ref }}=\frac{1}{\sqrt{3 b \rho}}, \quad \omega_{1}^{2}=1-a R_{1 A}, \quad \omega_{2}^{2}=1-a R_{1 B}, \\
\varepsilon_{1}=a \rho-\frac{R_{1 A}}{\rho}, \quad \varepsilon_{2}=a \rho-\frac{R_{1 B}}{\rho}, \quad \rho=\sqrt{\frac{L}{C}},
\end{gathered}
$$

$$
\begin{aligned}
\beta_{1}=R_{1 A} b V_{\text {ref }}^{2}, \quad \beta_{2} & =R_{1 B} b V_{\text {ref }}^{2}, \quad x=\frac{V_{1}}{V_{\text {ref }}}, \\
y & =\frac{V_{2}}{V_{\text {ref }}},
\end{aligned}
$$

the nondimensional circuit equation can be rewritten as follows:

$$
\begin{aligned}
& \ddot{x}-\left(\varepsilon_{1}-x^{2}\right) \dot{x}+\omega_{1}^{2} x+\beta_{1} x^{3}-\mu(\dot{y}-\dot{x})=0, \\
& \ddot{y}-\left(\varepsilon_{2}-y^{2}\right) \dot{y}+\omega_{2}^{2} y+\beta_{2} y^{3}-\mu(\dot{x}-\dot{y})=0,
\end{aligned}
$$

where the dot denotes the derivation with respect to the nondimensional time $t$. System (6a) and (6b) shows that the mathematical model describing the coupled van der PolDuffing electrical circuit is similar to the model defined in (1a) and (1b). Thus our motivation in this work is to carry out an abstract study of the system evolution (la) and (lb); thereafter, the results obtained can be exploited as predictions for the physical system.

\section{Stability Analysis}

3.1. Oscillation Quenching. In addition to the classical phase locking phenomenon, oscillation death also referred to as amplitude death or oscillation quenching is one of the most 


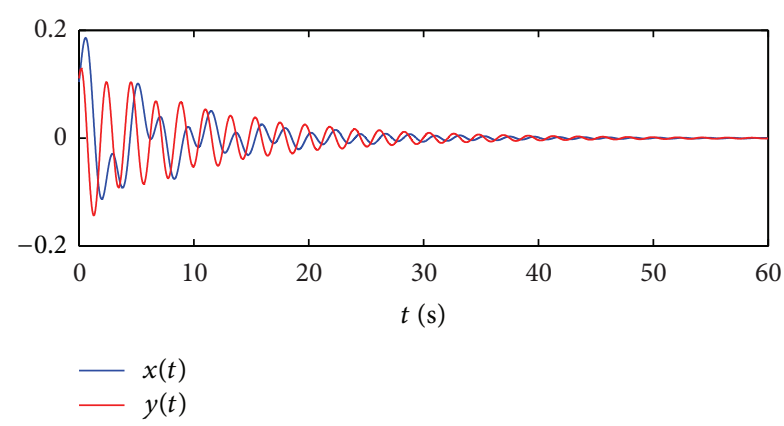

(a)

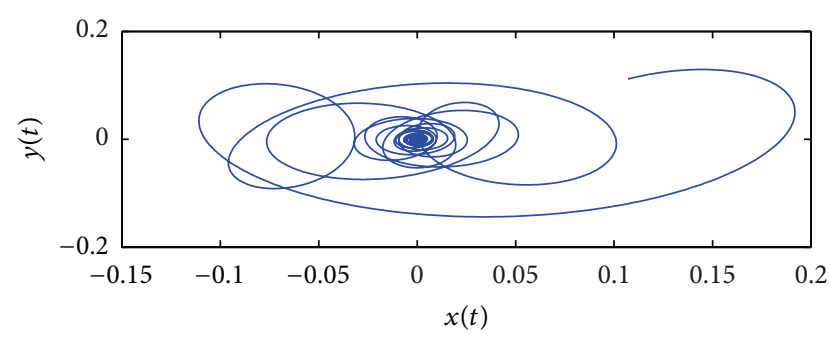

(b)

Figure 2: Time traces (a) and phase portraits (b) corresponding to a quenching behaviour of the mutually coupled van der Pol-Duffing oscillators. Amplitudes of both oscillators decay to zero as time evolves. The parameters are $\varepsilon=1.25 ; \beta=1.00 ; \mu=1.50 ; \Delta=10$.

intriguing effects that can occur in mutually coupled limit cycle oscillators when, due to coupling, oscillations stop completely $[6,21,22]$. Two kinds of oscillation death are distinguished. In the first type, the coupling creates a saddlenode pair of fixed points on the limit cycle of the entrained oscillations, while the second type is associated with a classical Hopf bifurcation in which the oscillation amplitudes are damped out up to reach a stable equilibrium/fixed point
$[6,21]$. In this section, conditions for the occurrence of amplitude death behaviour are derived by examining the stability of the trivial equilibrium point (i.e., the origin $\left.O=(0,0,0,0)^{T}\right)$ of the coupled system $(2 \mathrm{a})-(2 \mathrm{~d})$. In this regard, the Jacobian matrix evaluated at any equilibrium point $\left(x_{0}, u_{0}, y_{0}, v_{0}\right)^{T}$ can be easily computed as follows:

$$
M_{J}=\left[\begin{array}{cccc}
0 & 1 & 0 & 0 \\
-1-3 \beta x_{0}^{2}-2 u_{0} x_{0} & \varepsilon-\mu-x_{0}^{2} & 0 & \mu \\
0 & 0 & 0 & 1 \\
0 & \mu & -1-\Delta-3 \beta y_{0}^{2}-2 v_{0} y_{0} & \varepsilon-\mu-y_{0}^{2}
\end{array}\right]
$$

Thus the Jacobean matrix evaluated at the equilibrium point $O(0,0,0,0)^{T}$ satisfies the following characteristic equation $\left(\operatorname{det}\left(M_{J}-\lambda I_{d}\right)=0\right)$ :

$$
\lambda^{4}+c_{3} \lambda^{3}+c_{2} \lambda^{2}+c_{1} \lambda+c_{0}=0
$$

where

$$
\begin{gathered}
c_{0}=1+\Delta, \quad c_{1}=(2+\Delta)(\mu-\varepsilon), \\
c_{2}=\varepsilon^{2}-2 \mu \varepsilon+\Delta+2, \quad c_{3}=2(\mu-\varepsilon) .
\end{gathered}
$$

A set of necessary and sufficient conditions for all the roots of (8a) and (8b) to have negative real parts is given by the wellknown Routh-Hurwitz criterion expressed in the form

$$
\begin{gathered}
c_{i}>0 \quad(i=0,1,2,3), \\
c_{3} c_{2}-c_{1}>0, \\
c_{3}\left(c_{1} c_{2}-c_{0} c_{3}\right)-c_{1}^{2}>0 .
\end{gathered}
$$

After some algebraic manipulations, the region of parameters space corresponding to the regime of oscillation death behaviour (i.e., stable equilibrium point) is defined by the following inequality:

$$
\varepsilon<\mu<\frac{\varepsilon}{2}+\frac{\Delta^{2}}{4 \varepsilon(2+\Delta)} .
$$

In addition, $\Delta$ must be greater than the critical value given by

$$
\Delta_{c}=\varepsilon^{2}+\varepsilon \sqrt{4+\varepsilon^{2}} .
$$

If conditions (9a)-(9c) are not satisfied, the coupled system exhibits oscillatory motion; correspondingly the boundaries defined in (10a) and (10b) may be associated with a Hopf bifurcation as will be discussed in the next section. Typical phase portrait and time traces related to a quenching state of the coupled system are provided in Figure 2.

3.2. Hopf Bifurcation. The Hopf type bifurcations [23] are observed if the following conditions are fulfilled: (a) $(8 \mathrm{a})$ and $(8 \mathrm{~b})$ have a pair of pure imaginary complex eigenvalues $\lambda_{1,2}= \pm j \omega_{0}= \pm j \sqrt{1+\Delta / 2}$; (b) the derivative of the real part of $\lambda$ with respect to the control parameter must be different to zero when this control parameter approaches the critical value (i.e., $d \lambda /\left.d \alpha\right|_{\mu=\mu_{c}} \neq 0 ; \mu$ is the bifurcation control parameter and $\mu_{c}$ is a solution of the equation $\left.\operatorname{Re}(\lambda)=0\right)$. The derivative of the real part of $\lambda$ versus the control parameter $\mu$ can be found from (8a) and reads $\left.\operatorname{Re}(d \lambda / d \mu)\right|_{\mu=\mu_{c}}=\varepsilon(2+$ $\Delta)(\mu-\varepsilon) /\left((\mu-\varepsilon)^{2}(4+2 \Delta)+\varepsilon^{2}(\varepsilon-2 \mu)^{2}\right)$. It is clear that this quantity is different to zero, since $\mu>\varepsilon$ provided that $\mu$ approaches the critical value $\mu_{c}=\varepsilon / 2+\Delta^{2} / 4 \varepsilon(2+\Delta)$ 


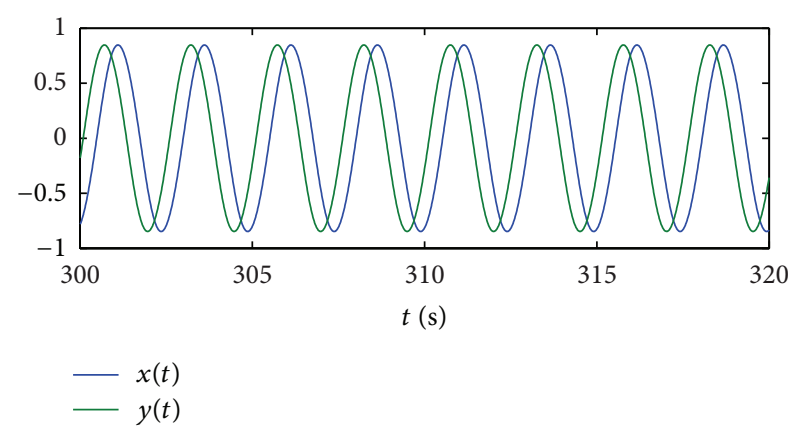

(a)

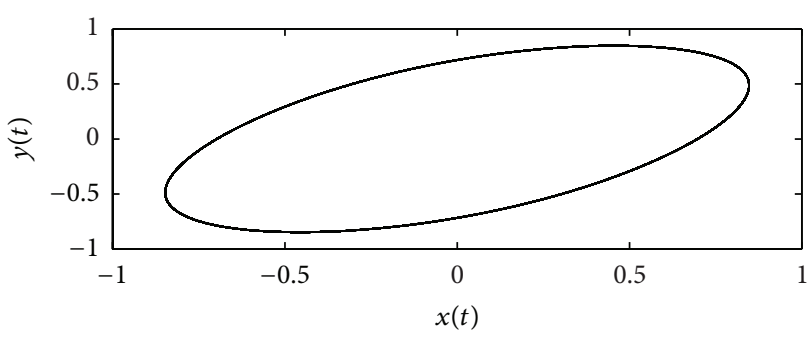

(b)

Figure 3: Time traces (a) of the limit cycle (b) born after the Hopf bifurcation showing a quasi-sinusoidal behavior of the system. The parameters are $\varepsilon=1.25 ; \beta=0.50 ; \mu=2.40 ; \Delta=10$.

corresponding to the upper bound of inequality (10a). Note that the lower bound of the same inequality is associated with a degenerate Hopf bifurcation. Briefly recall that when the Hopf bifurcation occurs, the coupled system moves from the state of amplitude death to an oscillatory regime. For illustrating purposes, we provide in Figure 3 the time traces and phase portrait corresponding to a quasi-sinusoidal behavior of the system after the occurrence of the Hopf bifurcation. Furthermore, in order test the validity of our analytical results, system (2a)-(2d) is integrated numerically following the Runge-Kutta scheme and the local maxima of solutions (i.e., $x(t)$ and $y(t))$ are plotted versus the coupling strength $\mu$. The results presented in Figure 4 are consistent with the analytical formulas (i.e., (10a) and (10b)) proposed for the definition of the parameter space corresponding to the quenching of oscillations in the coupled system. Note that, with the parameters setting in Figure 4, no quenching phenomenon is observed for values of $\Delta$ lower than the critical value $\Delta_{c}=4.1981$. Also note, from Figure 4, the increase of the quenching domain/zone with increasing $\Delta$.

\section{Routes to Chaos}

According to the results obtained by Kuznetsov and coworkers [18-20], the dynamics of the coupled van der Pol-Duffing system with respect to the parameters $\varepsilon, \mu$, and $\Delta$ is well mastered. We now concentrate on the effects of the cubic nonlinearity (i.e., parameter $\beta$ ) on the dynamics of the system. For this purpose, system (2a)-(2d) is integrated numerically to reveal different scenarios/routes to chaos in our model using the classical fourth-order Runge-Kutta formulas. For each value of $\beta$, the system is integrated for a sufficiently long time and transient is discarded. The bifurcation diagrams are obtained by plotting the maxima of $x(t)$ and $y(t)$ in terms of the control parameter $\beta$. The integration time step is always kept $\Delta t \leq 0.005$ and computations are carried out using variables and constants parameters in extended mode. To evaluate the degree of chaos, we compute the largest numerical one dimensional (1D) Lyapunov exponent (LLE), (LLE $>0$ for chaotic states, LLE $<0$ for regular states) and draw the corresponding bifurcation diagram. The largest numerical one dimensional (1D) Lyapunov is expressed as

$$
\begin{aligned}
& \text { LLE }=\lim _{t \rightarrow \infty}\left[\left(\frac{1}{t}\right) \ln (d(t))\right] \\
& \text { with }\left(d(t)=\sqrt{\delta x^{2}+\delta u^{2}+\delta y^{2}+\delta v^{2}}\right)
\end{aligned}
$$

and computed from the variational equations obtained by perturbing the solutions of (2a)-(2d) as follows: $x \rightarrow x+\delta x$, $u \rightarrow u+\delta u, y \rightarrow y+\delta y$, and $v \rightarrow v+\delta v$ with the help of the algorithm proposed by Wolf et al. in [24]. Sample results are shown in Figure 5 from which a very rich and striking dynamic behaviour can be seen when the bifurcation control parameter $\beta$ is slowly monitored. The bifurcation structures include period-doubling, symmetry-breaking, and small windows of regularity in chaotic domains. Those bifurcation scenarios are perfectly traced by the Lyapunov spectra (see Figure 5). Typical phase portraits showing sample states of the coupled system are shown in Figure 6. The phase portraits of Figures 6(a)-6(d) correspond to regular states while the one shown in Figure 6(d) depicts a chaotic behavior of the system. Note that attractors in Figures 6(b) and 6(c) are asymmetric and correspondingly coexisting solutions are obtained by changing the initial conditions. This denotes the bistability property of the coupled system. Similarly, the extreme sensitivity of the coupled system with respect to the coupling constant $\mu$ is illustrated by the bifurcation diagrams provided in Figures 7(a) and 7(b). It is worth mentioning that the results obtained here complement previous work presented in the pioneering literature [18-20].

\section{Analog Computation}

The analog simulation method is a convenient strategy to scan the parameter range in order to find the proper parameter values for a numerical integration. In contrast to numerical computation, there is no need to wait for long transient times when performing an analog computation. This may serve to explain the increasing interest devoted to this type of implementation for the analysis of complex nonlinear systems [2527]. The aim of this section is to design and implement 

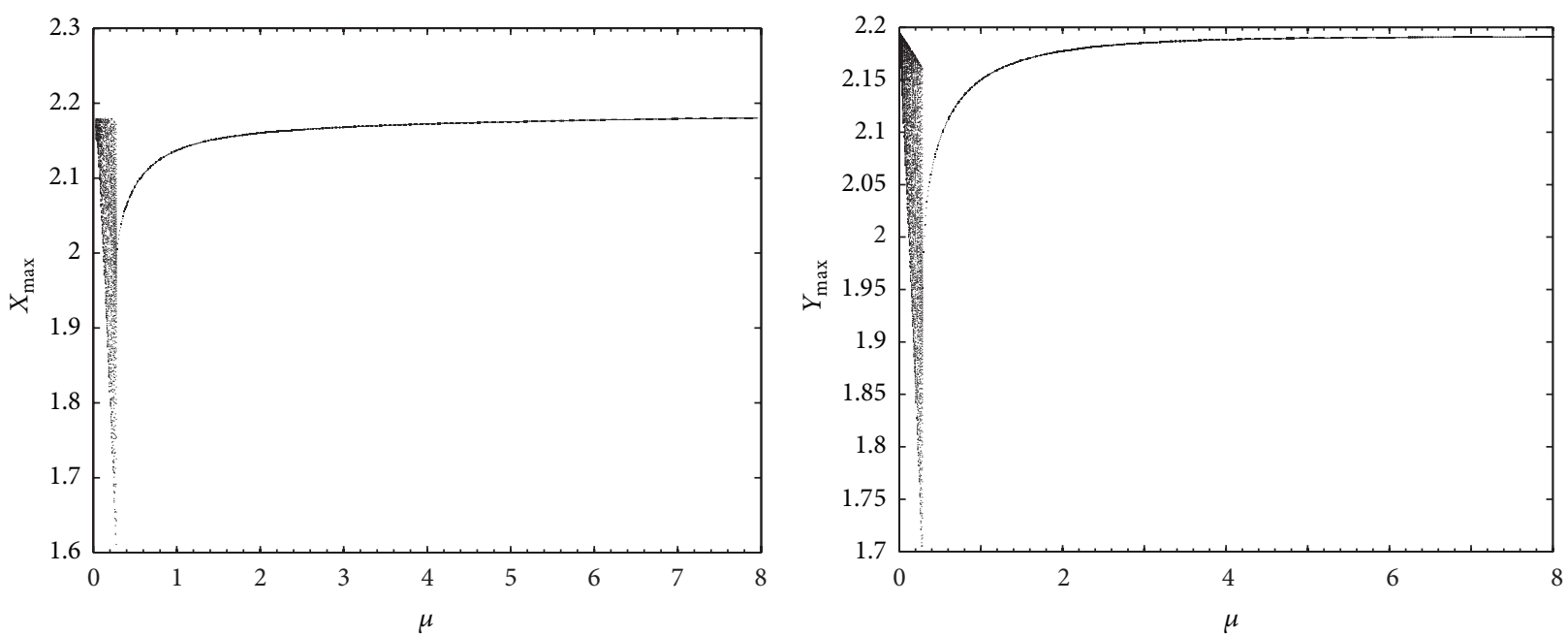

(a)
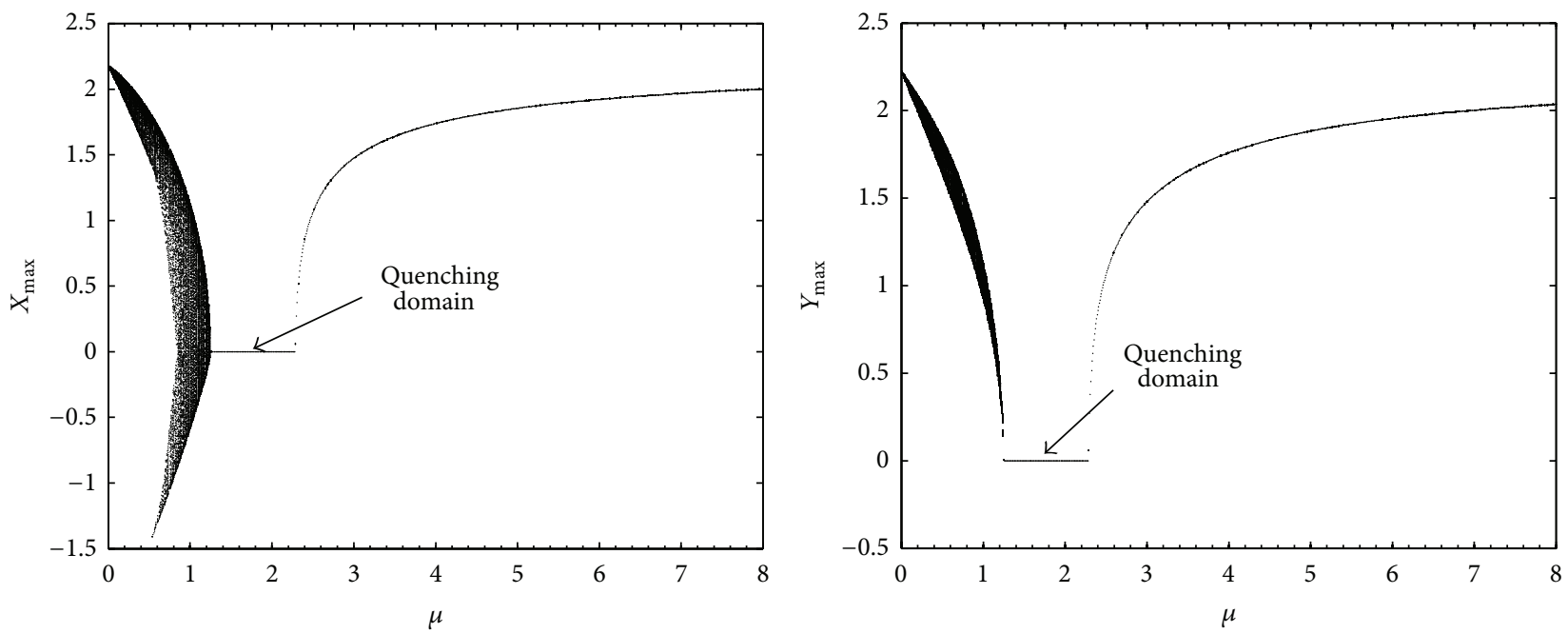

(b)
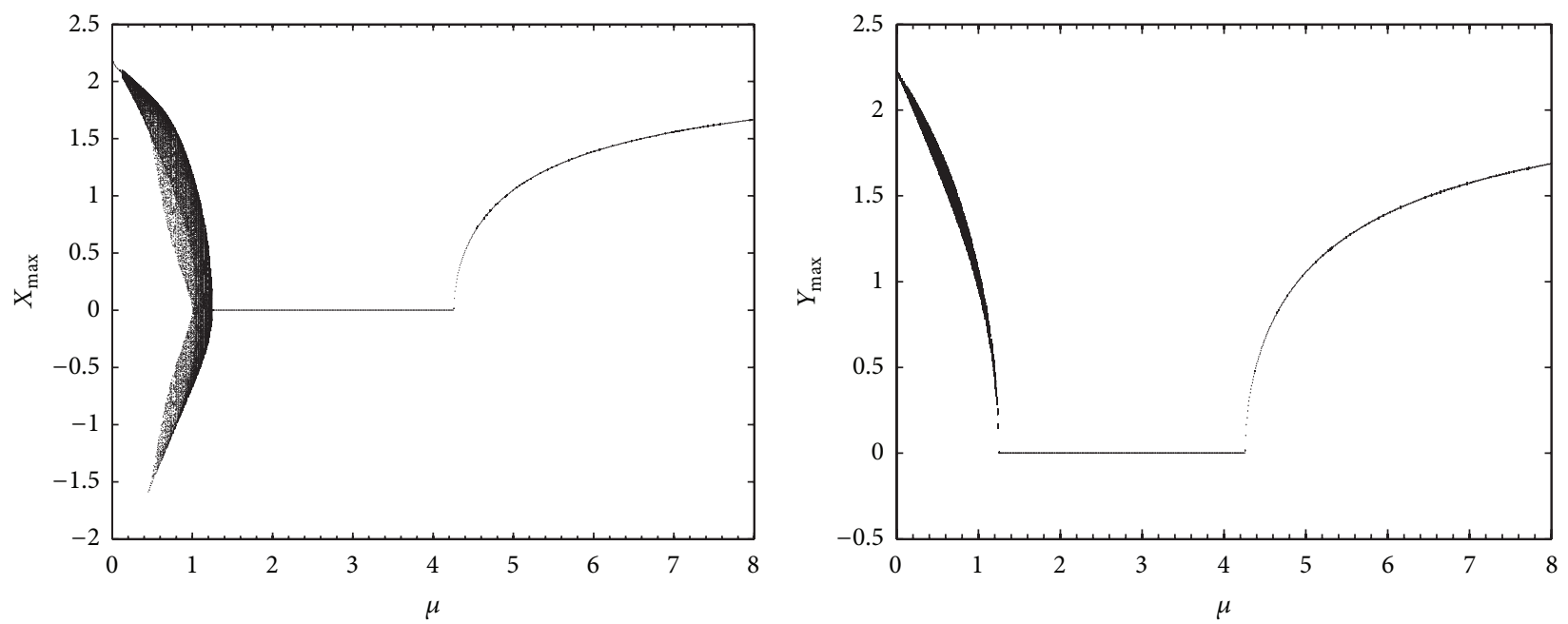

(c)

FIGURE 4: Amplitude death zone as a function of the coupling strength $\mu$ for three different values of frequency detuning $\Delta$ : (a) no death zone is observed for $\Delta=1$; (b) smaller death zone is observed for $\Delta=10$; (c) larger death zone is observed for $\Delta=20$. The rest of parameters are $\beta=0.50 ; \varepsilon=1.25$. 


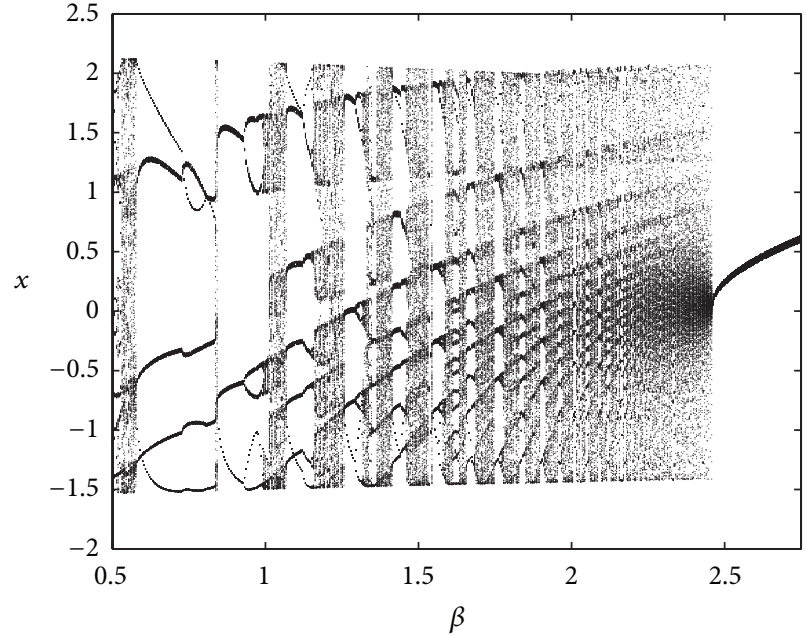

(a)

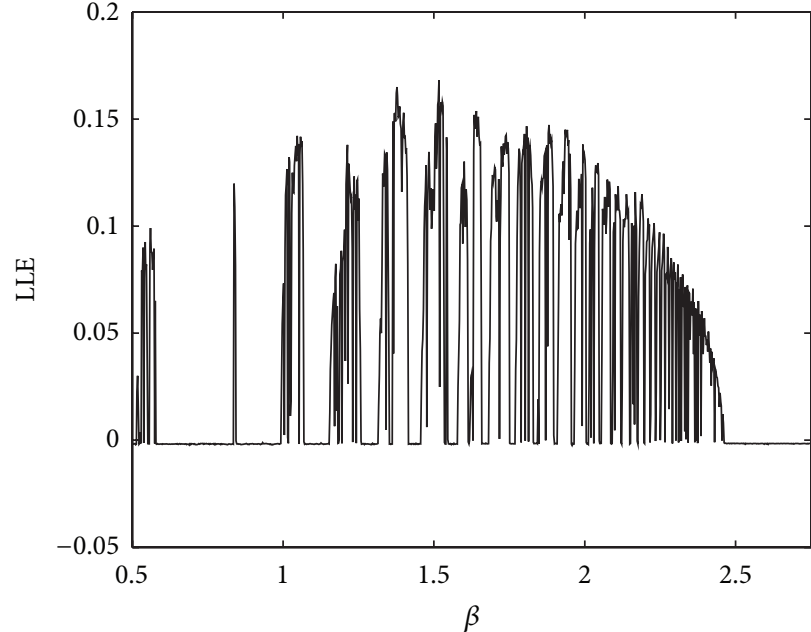

(b)

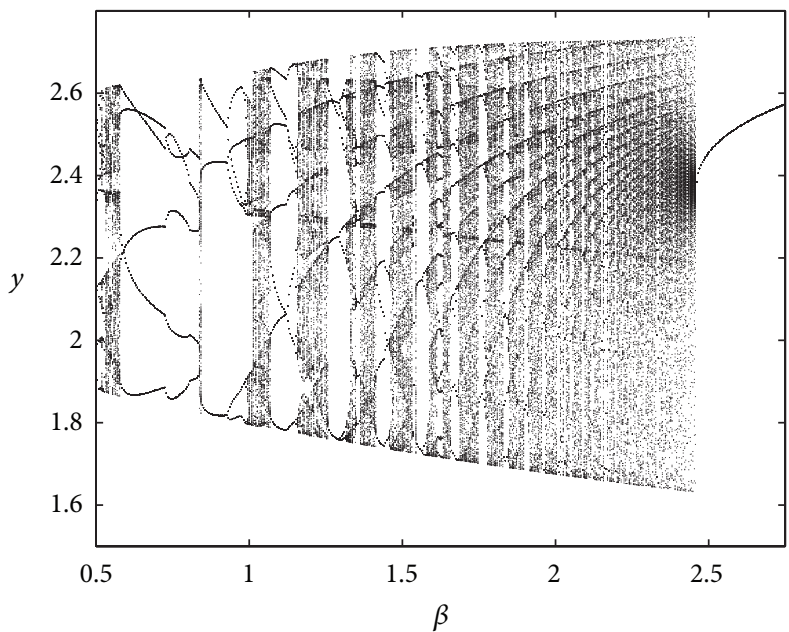

(c)

FIGURE 5: Bifurcation diagram of the system showing the coordinates $x$ (a) and $y(\mathrm{c})$ in terms of the control parameter $\beta$ and the corresponding graph (c) of the largest 1D Lyapunov exponent (LLE). The rest of parameters are $\varepsilon=2.50 ; \mu=1.25 ; \Delta=10$.

an appropriate analog circuit/simulator for the analysis of the model defined by the set of (1a) and (1b) in order to verify and complement the theoretical results obtained previously. The scenarios/routes to chaos in the system are searched experimentally. Numerical and experimental phase portraits are compared.

5.1. Design of the Analog Simulator. Figure 8 is a circuit diagram of the proposed electronic simulator. The electronic multipliers $M_{i}(i=1,2,3,4,5,6)$ are the analog devices AD633JN versions of the AD633 four-quadrant voltage multiplier chips. They are used to implement the nonlinear terms of our model. The integrators are built by using operational amplifiers (TL084CN) with feedback capacitors while summations are obtained using operational amplifiers with feedback resistors and input resistors. Adopting an appropriate time scaling [25-27], the simulator outputs can directly be displayed on an oscilloscope by connecting the voltages to the $X$ input and $Y$ input. For the oscillator $X$ (resp., $Y)$ the phase portraits are obtained by feeding the output of $U_{1} C$ (resp., $U_{5} D$ ) to the $X$ input and that of $U_{1} A$ (resp., $U_{5} B$ ) to the $Y$ input. It can be shown that the voltages at $x$ (output of $U_{1} C$ ) and $y$ (output of $U_{5} D$ ) satisfy the set of coupled nonlinear differential equations (1a) and (1b). In terms of electronic circuit components, the parameters of (1a) and (1b) are expressed as follows:

$$
\begin{aligned}
\varepsilon & =\frac{1}{10^{4} R_{15} C_{12}}=\frac{1}{10^{4} R_{25} C_{22}}, \\
\beta & =\frac{1}{10^{10} R_{13} R_{11} C_{11} C_{12}}=\frac{1}{10^{10} R_{23} R_{21} C_{21} C_{22}}, \\
\mu & =\frac{1}{10^{4} R_{17} C_{12}}=\frac{1}{10^{4} R_{27} C_{22}}, \\
\Delta+1 & =\frac{1}{10^{8} R_{26} R_{21} C_{21} C_{22}} .
\end{aligned}
$$



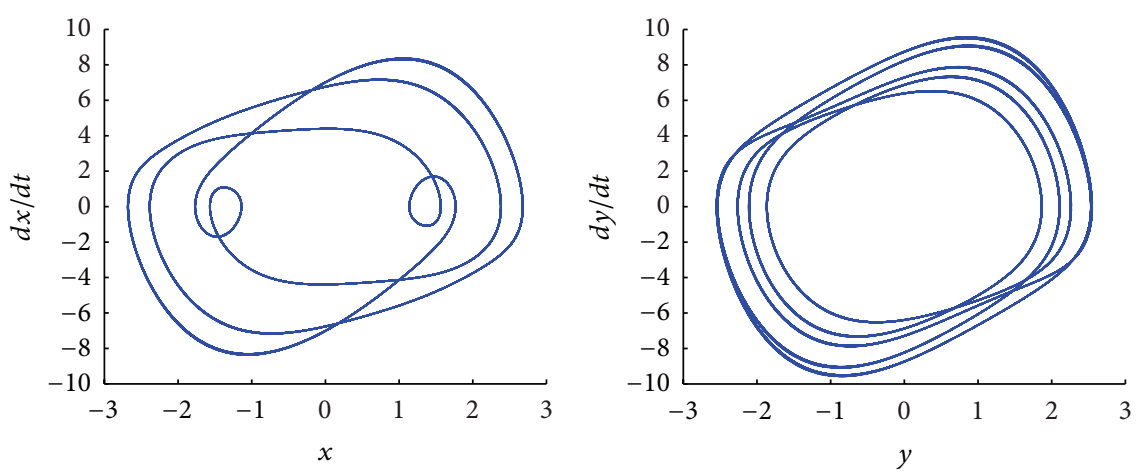

(a)
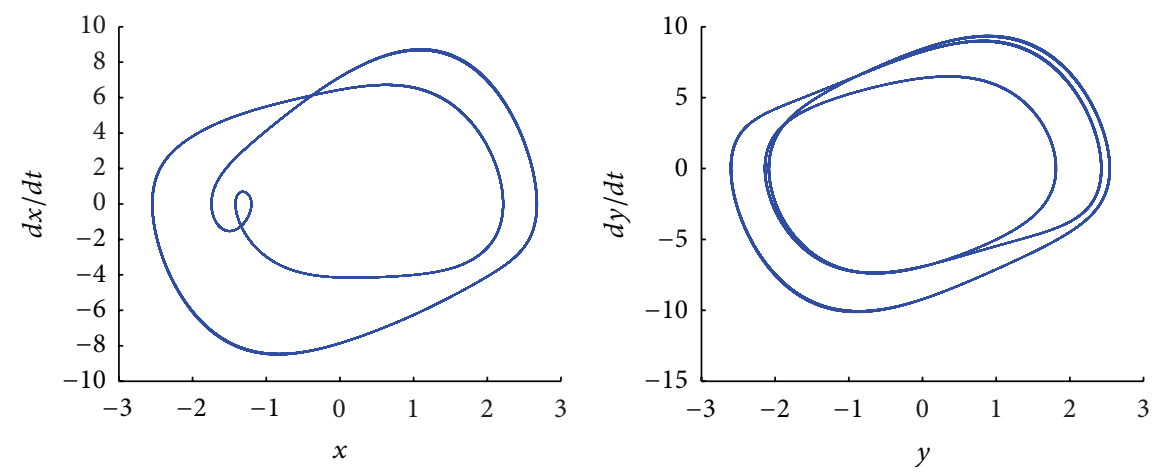

(b)
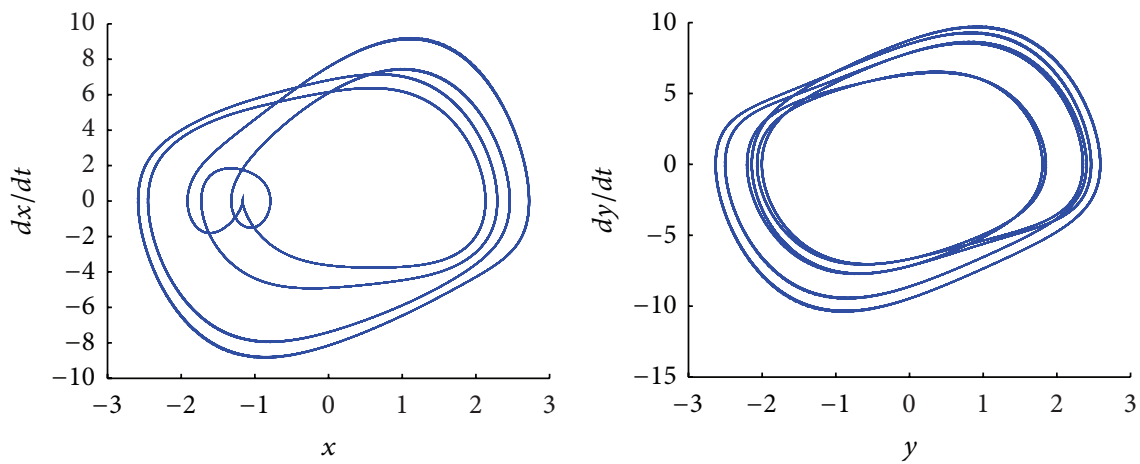

(c)
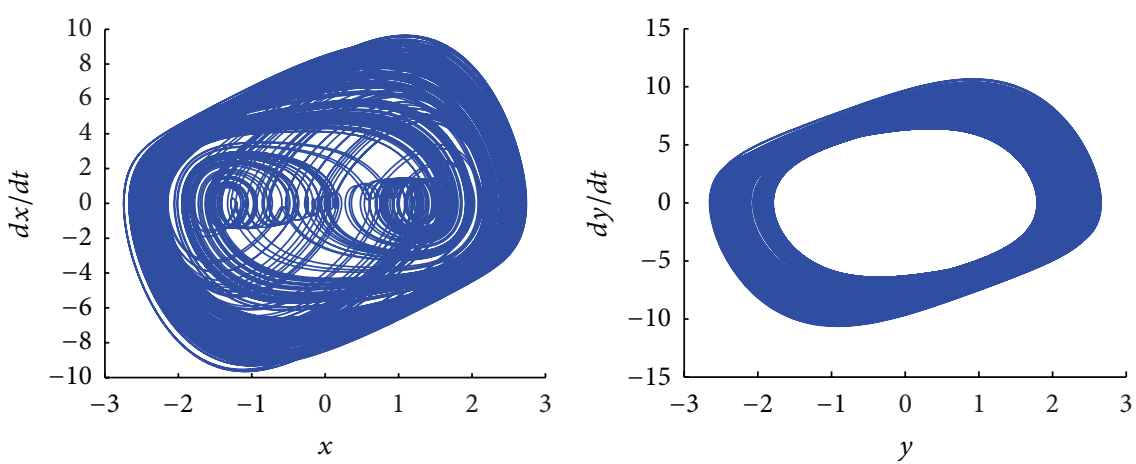

(d)

FIGURE 6: Numerical phase portraits of the coupled system showing typical dynamic states for (a) period-5 for $\beta=0.65$; (b) period-3 for $\beta=0.90$; (c) period -6 for $\beta=0.95$; (d) chaos for $\beta=1.05$. The rest of parameters are those in Figure 5 . 


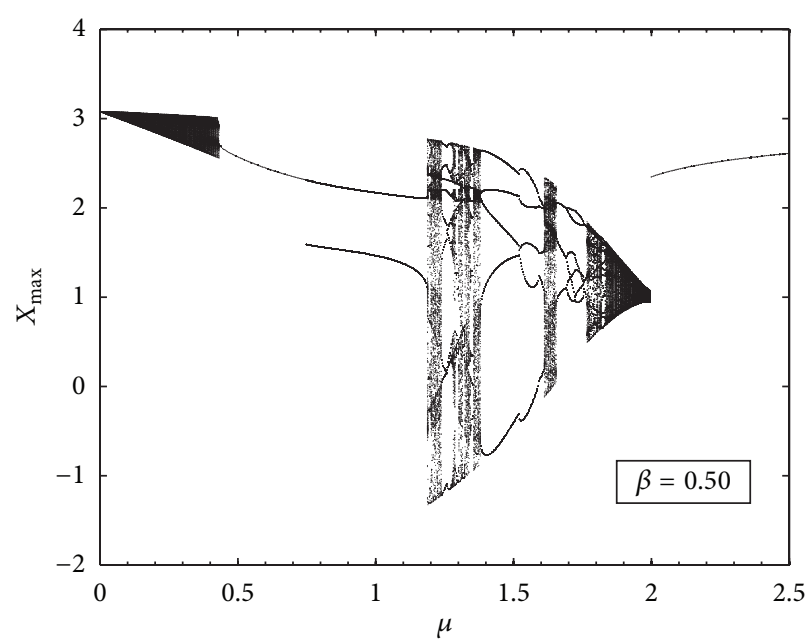

(a)

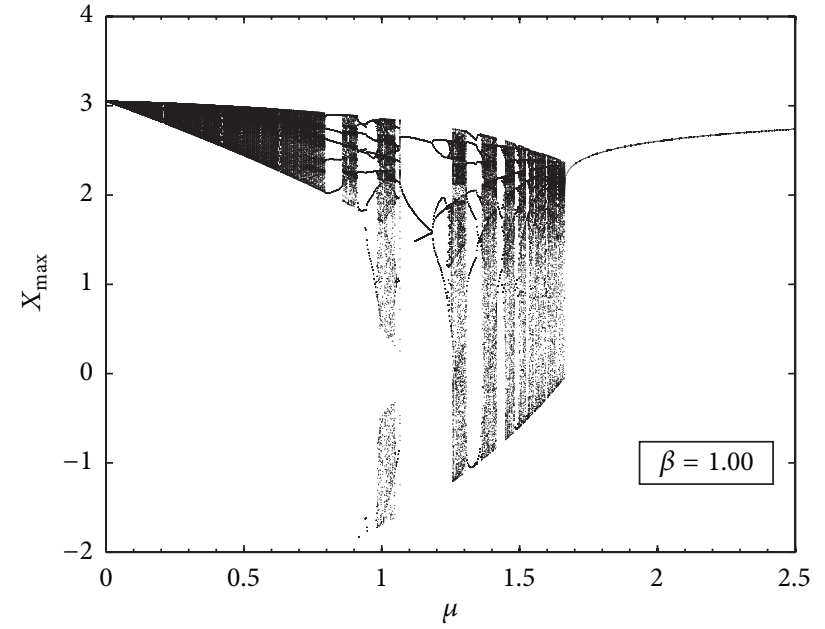

(b)

FIGURE 7: One parameter bifurcation diagrams showing the coordinate $X$ in the Poincare cross section versus the coupling constant $\mu$ for different values of $\beta$ (i.e., $\beta=0.50$ (a) and $\beta=1.00(\mathrm{~b})$ ). The rest of parameters are $\varepsilon=2.50 ; \Delta=10$.

These mathematical definitions take into account the following critical relationships between the values of resistors and capacitors:

$$
\begin{gathered}
10^{6} R_{14} C_{12}=10^{6} R_{24} C_{22}=1, \\
10^{8} R_{16} R_{11} C_{11} C_{12}=1 .
\end{gathered}
$$

The time unit adopted is $10^{-4} \mathrm{~s}$. Mention that the time scaling process offers to the analog devices the possibility to operate under their bandwidth. Furthermore the time scaling offers the possibility to simulate the behaviour of the system at any given frequency by expressing the real time variable $t$ versus the analog computation time variable $\tau\left(t=10^{-a} \tau\right)$ allowing the simulation frequency to be $10^{+a}$ times less than the real frequency. Here, $a$ is a positive integer depending on the values of resistors and capacitors involved in the analog simulation. The reader is referred to the work of Chedjou et al. [26] for the complete derivation procedure. The values of circuit's components of the overall analog simulator are provided in Table 1. From (12a) and (12b), it is possible to control each system parameter by simply monitoring a single resistor or a pair of resistors simultaneously. For instance, $\Delta$ can be controlled with $R_{26}$ and $\varepsilon, \beta, \mu$ with $R_{j 5}, R_{j 3}$, and $R_{j 7}(j=1,2)$, respectively. A photograph of the experimental analog simulator is shown in Figure 9.

5.2. Quenching Phenomenon. In order to experimentally observe the quenching phenomenon in the coupled van der Pol-Duffing oscillators considered in present paper, the values of resistor $R_{26}$ have been successively chosen as indicated in the first column of Table 2, leading to the following settings of parameter $\Delta: 7.5,10,15$, and 20, respectively. As in Section 3, we also set the nonlinearity parameter to $\beta=0.50$ by choosing $R_{j 3}(j=1,2)=200 \Omega$ while the values of control resistors $R_{j 5}$ are $R_{j 5}(j=1,2)=8 \mathrm{k} \Omega$ leading to $\varepsilon=1.25$. For each value of $R_{26}$, the pair of resistors $R_{j 7}$ (i.e., parameter $\mu$ )
TABLE 1: Values of capacitors and resistors used in the overall electronic simulator.

\begin{tabular}{lcc}
\hline Resistors $(\Omega)$, capacitors $(\mathrm{nF})$ & Oscil. $X(j=1)$ & Oscil. $Y(j=2)$ \\
\hline$R_{j 1}$ & $10^{4}$ & $10^{4}$ \\
$R_{j 2}$ & $10^{4}$ & $10^{4}$ \\
$R_{j 3}$ & $0-10^{3}$ & $0-10^{3}$ \\
$R_{j 4}$ & 100 & 100 \\
$R_{j 5}$ & $0-10^{4}$ & $0-10^{4}$ \\
$R_{j 6}$ & $10^{4}$ & $0-10^{4}$ \\
$R_{j 7}$ & $0-10^{4}$ & $0-10^{4}$ \\
$C_{j 1}$ & 10 & 10 \\
$C_{j 2}$ & 10 & 10 \\
$R_{31}$ (coupling) & $10^{4}$ & $10^{4}$ \\
\hline
\end{tabular}

TABLE 2: Values of parameters of the system for quenching phenomena; subscript $j=1,2$ for oscillators $X$ and $Y$, respectively; all resistors values are expressed in $\Omega$.

\begin{tabular}{lcccc}
\hline $\begin{array}{l}\text { Detuning } \\
\text { resistor }\end{array}$ & \multicolumn{2}{c}{ Theoretical values of $R_{j 7}$} & \multicolumn{2}{l}{ Experimental values of $R_{j 7}$} \\
$R_{26}$ & $R_{j 7 \text { min }}$ & $R_{j 7 \text { max }}$ & $R_{j 7 \text { min }}$ & $R_{j 7 \text { max }}$ \\
\hline 1176 & 5527 & $8 \cdot 10^{3}$ & 5530 & 8012 \\
909 & 4362 & $8 \cdot 10^{3}$ & 4373 & 8004 \\
625 & 3056 & $8 \cdot 10^{3}$ & 3065 & 7990 \\
476 & 2346 & $8 \cdot 10^{3}$ & 2352 & 8015 \\
\hline
\end{tabular}

is slowly monitored. The experiments indicate that when $R_{j 7}$ varies inside the interval $\left[R_{j 7 \text { min }}, R_{j 7 \text { max }}\right]$, the two coupled oscillators stop oscillating. This nonoscillating state is marked by the presence of a point on the oscilloscope screen in $X Y$ mode. The experimental values of the thresholds $R_{j 7 \text { min }}$ and $R_{j 7 \text { max }}$, inside which the quenching phenomenon is observed, are indicated in the two last columns of Table 2. 


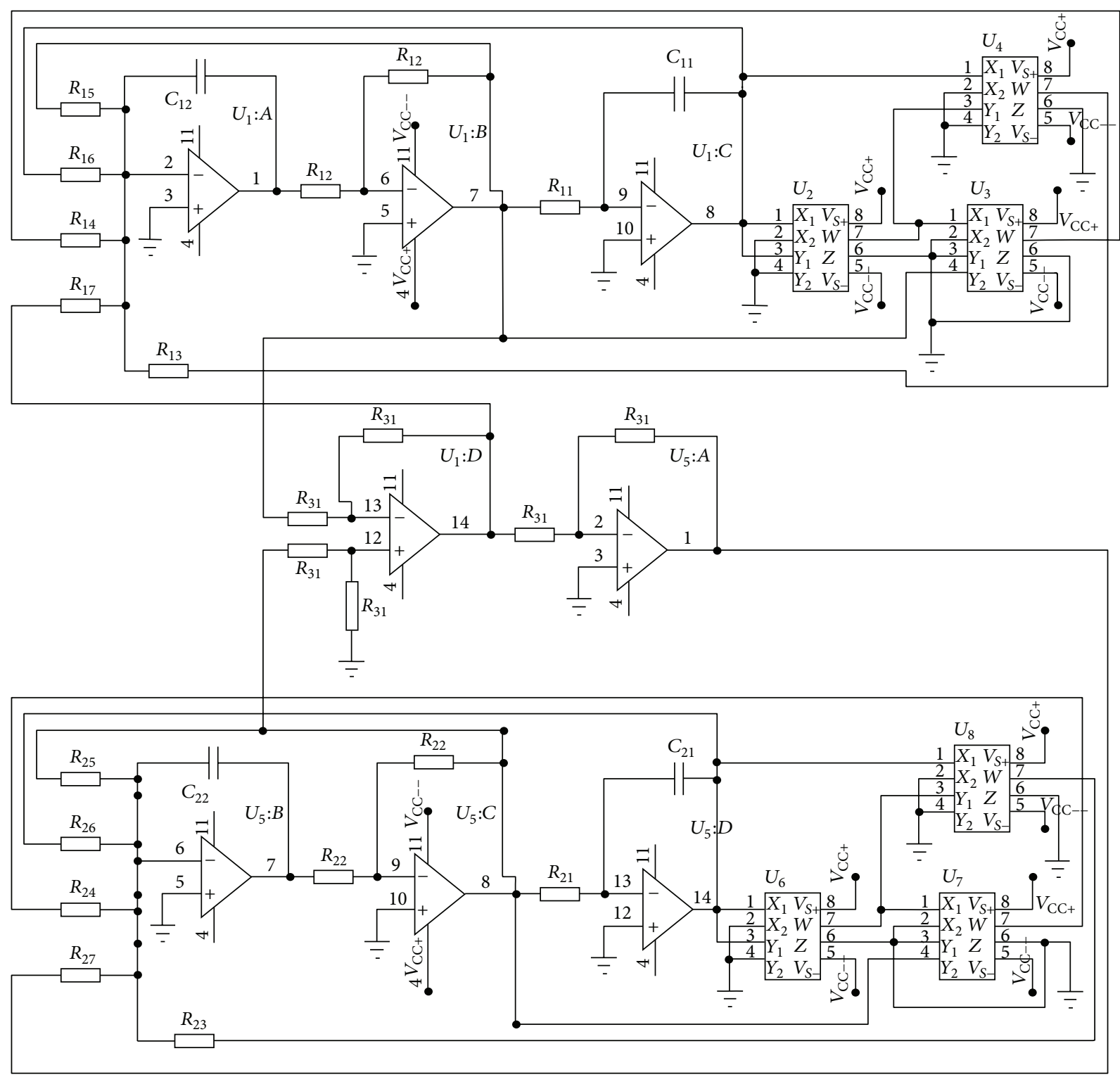

FIGURE 8: Schematic diagram of the electronic simulator. The network built around op. amplifiers U1D and U5A is the coupling module while the upper and lower networks implement oscillators $X$ and $Y$, respectively. The values of capacitors and resistors are those in Table 1.

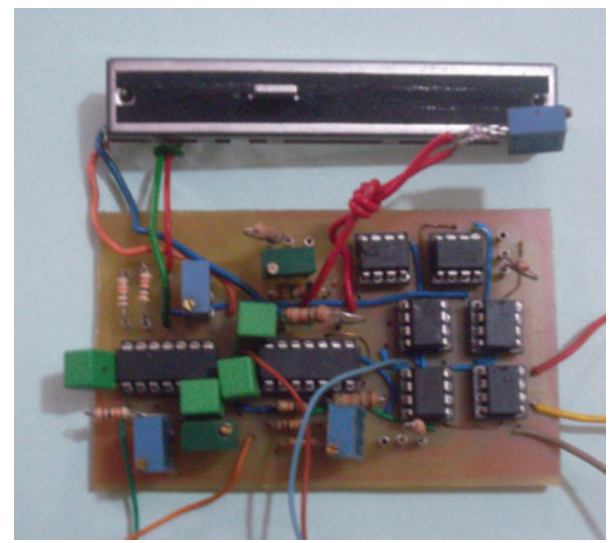

FIGURE 9: Photograph of the experimental analog simulator.
Those values are well in agreement with theoretical ones presented in the second and third columns of the same table. It is important to stress that, for values of $R_{26} \geq 1942 \Omega$, the system keeps oscillating (i.e., no quenching is observed) for any choice of the control resistors $R_{j 7}$. This observation is also in agreement with the theoretical analysis (see Section 3 ) from which the predicted critical value of $R_{26}$ under which the quenching phenomenon may occur is $R_{26 c}=1923 \Omega$.

5.3. Bifurcation and Chaos. We now focus on the experimental bifurcation study of the coupled van der Pol-Duffing system with the help of the electronic simulator. The effects of cubic nonlinearity (i.e., parameter $\beta$ ) on the behavior of the coupled system are investigated by monitoring the pair of resistors $R_{j 3}(j=1,2)$ while keeping the rest of electronic 

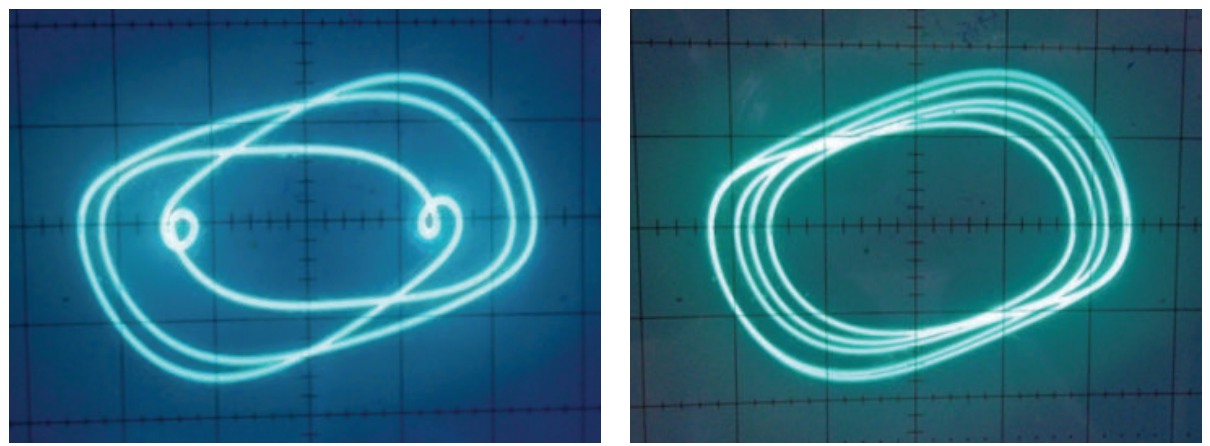

(a)
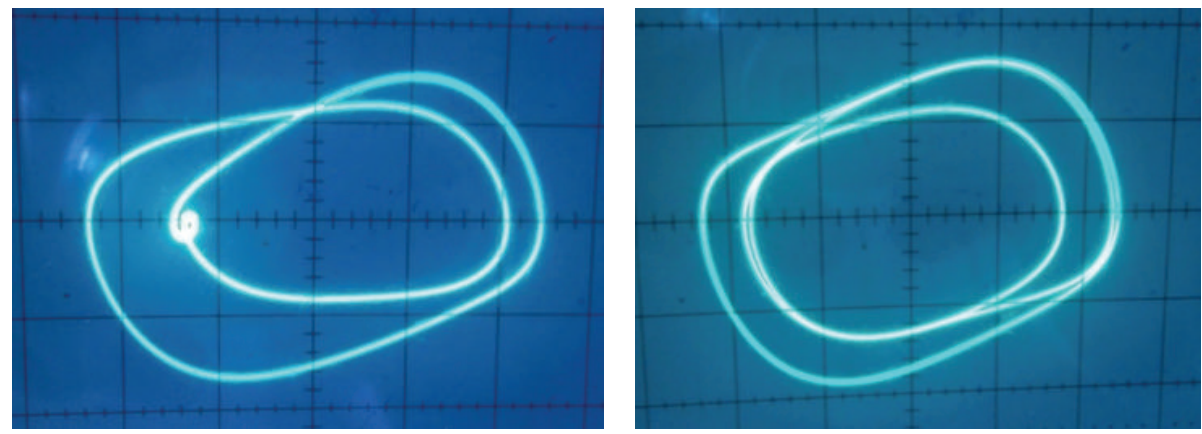

(b)
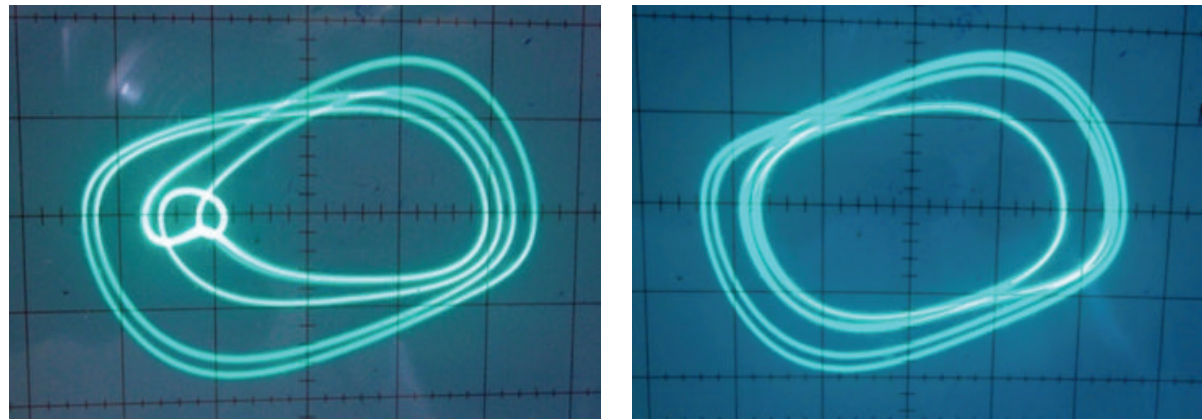

(c)
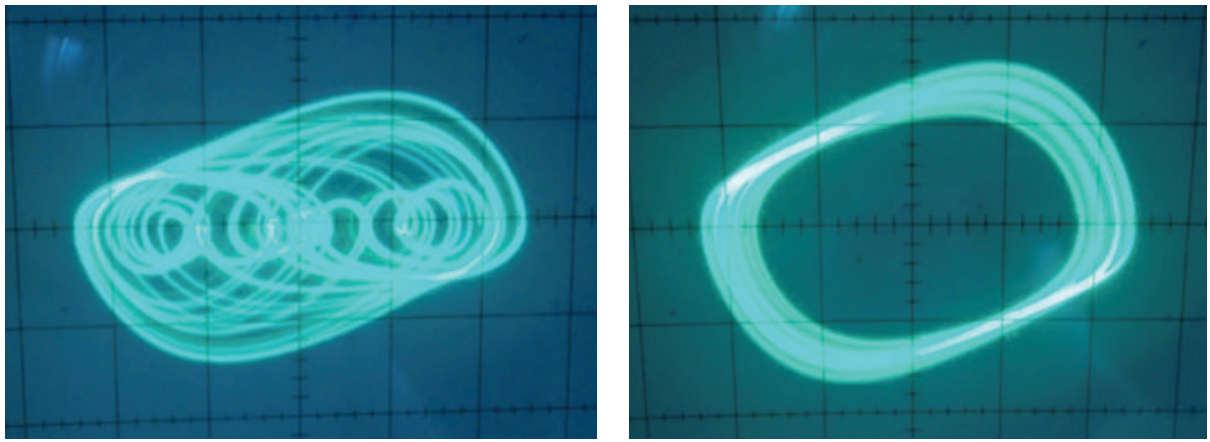

(d)

FIGURE 10: Experimental results showing typical phase portraits of the coupled system: (a) period -5 for $R_{j 3}=155 \Omega$; (b) period-3 for $R_{j 3}=$ $110 \Omega$; (c) period-6 for $R_{j 3}=105 \Omega$; (d) chaos for $R_{j 3}=95 \Omega$. For all pictures displayed, the scales are $x=1 v /$ div and $y=5 v /$ div. 
components values constant (see Table 1). In this section, the values of control resistors are chosen as follows: $R_{26}=$ $909 \Omega, R_{j 7}(j=1,2)=4 \mathrm{k} \Omega$, and $R_{j 7}(j=1,2)=8 \mathrm{k} \Omega$. The choice of these values is justified by our wish to use the same sets of parameters for both numerical and experimental analyses (i.e., $\varepsilon=2.50, \mu=1.25$, and $\Delta=10$ ). When monitoring simultaneously the control resistors $R_{j 3}$, it is found that the electronic simulator experiences a rich and striking dynamical behaviour. Some sample phase portraits obtained experimentally are shown in Figure 10. Note the similarity between the numerically computed phase portraits (see Figure 6) and the experimental ones. Furthermore, from Figure 10, one can see that the experimental circuit experiences the same bifurcation structures as those obtained numerically.

\section{Concluding Remarks}

The main concern of this paper was to investigate the dynamics of two mutually coupled van der Pol-Duffing oscillators. Standard nonlinear dynamic tools including phase portraits, frequency spectra, Lyapunov exponent, and bifurcation diagrams were used as indicators of chaotic motion. We have found that the coupled system can experience a variety of bifurcations, namely, symmetry-breaking, period-doubling, crises, and Hopf bifurcation, when monitoring the cubic nonlinearity coefficient that accounts for the anisochronism of small oscillations. Furthermore, oscillation death behaviour as well as phase synchronization between the two oscillators was also examined. An appropriate analog simulator was constructed and employed for the investigations. Despite the tolerance on the values of electronic components and the imperfections of the op. amplifiers of the analog simulator, the experimental and theoretical results show a close agreement.

An interesting question under consideration is the study of the effects of both elastic and dissipative coupling on the dynamics of the coupled van der Pol-Duffing oscillators for more generalization. Another interesting issue under consideration is the analysis of the effects of time delay on the dynamics and synchronization of the coupled anisochronous oscillators in spite of practical coupling conditions and possible applications.

\section{Conflict of Interests}

The authors declare that there is no conflict of interests regarding the publication of this paper.

\section{References}

[1] A. Pikovsky, M. Rosenblum, and J. Kurths, Synchronization: A Universal Concept in Nonlinear Sciences, Cambridge University Press, Cambridge, UK, 2001.

[2] J. M. Gonzalez-Miranda, Synchronization and Control of Chaos: An Introduction for Scientists and Engineers, Imperial College Press, London, UK, 2004.

[3] B. van der Pol, "On relaxation-oscillations," The London, Edinburgh, and Dublin Philosophical Magazine and Journal of Sciences Series, vol. 7, no. 2, pp. 978-992, 1926.
[4] R. Yamapi and P. Woafo, "Dynamics and synchronization of coupled self-sustained electromechanical devices," Journal of Sound and Vibration, vol. 285, no. 4-5, pp. 1151-1170, 2005.

[5] H. B. Fotsin and P. Woafo, "Adaptive synchronization of a modified and uncertain chaotic van der Pol-Duffing oscillator based on parameter identification," Chaos, Solitons and Fractals, vol. 24, no. 5, pp. 1363-1371, 2005.

[6] A. Balanov, N. Janson, D. Postnov, and O. Sosnovtseva, Synchronization: From Simple to Complex, Springer, Berlin, Germany, 2009.

[7] M. Belhaq and A. Fahsi, "Frequency-locking in nonlinear forced oscillators near 3:1 and 4:1 resonances," Annals of Solid and Structural Mechanics, vol. 4, no. 1-2, pp. 15-23, 2012.

[8] A. P. Kuznetsov, E. P. Seleznev, and N. V. Stankevich, "Nonautonomous dynamics of coupled van der Pol oscillators in the regime of amplitude death," Communications in Nonlinear Science and Numerical Simulation, vol. 17, no. 9, pp. 3740-3746, 2012.

[9] Y. P. Emelianova, A. P. Kuznetsov, I. R. Sataev, and L. V. Turukina, "Synchronization and multi-frequency oscillations in the low-dimensional chain of the self-oscillators," Physica D: Nonlinear Phenomena, vol. 244, no. 1, pp. 36-49, 2013.

[10] Y. Zhai, I. Z. Kiss, and J. L. Hudson, "Amplitude death through a hopf bifurcation in coupled electrochemical oscillators: experiments and simulations," Physical Review E, vol. 69, no. 2, Article ID 026208, 2004.

[11] I. Ozden, S. Venkataramani, M. A. Long, B. W. Connors, and A. V. Nurmikko, "Strong coupling of nonlinear electronic and biological oscillators: reaching the "amplitude death" regime," Physical Review Letters, vol. 93, no. 15, Article ID 158102, 2004.

[12] D. W. Storti and R. H. Rand, "Dynamics of two strongly coupled van der Pol oscillators," International Journal of Non-Linear Mechanics, vol. 17, no. 3, pp. 143-152, 1982.

[13] T. Chakraborty and R. H. Rand, "The transition from phase locking to drift in a system of two weakly coupled van der pol oscillators," International Journal of Non-Linear Mechanics, vol. 23, no. 5-6, pp. 369-376, 1988.

[14] I. Pastor, V. M. Pérez-García, F. Encinas, and J. M. Guerra, "Ordered and chaotic behavior of two coupled van der Pol oscillators," Physical Review E, vol. 48, no. 1, pp. 171-182, 1993.

[15] M. Poliashenko, S. R. McKay, and C. W. Smith, "Hysteresis of synchronous-asynchronous regimes in a system of two coupled oscillators," Physical Review A, vol. 43, no. 10, pp. 5638-5641, 1991.

[16] M. Poliashenko, S. R. McKay, and C. W. Smith, "Chaos and nonisochronism in weakly coupled nonlinear oscillators," Physical Review A, vol. 44, no. 6, pp. 3452-3456, 1991.

[17] I. Pastor-Díaz and A. López-Fraguas, "Dynamics of two coupled van der Pol oscillators," Physical Review E, vol. 52, no. 2, pp. 1480-1489, 1995.

[18] A. P. Kuznetsov and J. P. Roman, "Synchronization of coupled anisochronous auto-oscillating systems," Nonlinear Phenomena in Complex Systems, vol. 12, no. 1, pp. 54-60, 2009.

[19] A. P. Kuznetsov and J. P. Roman, "Properties of synchronization in the systems of non-identical coupled van der Pol and van der Pol-Duffing oscillators. Broadband synchronization," Physica D: Nonlinear Phenomena, vol. 238, no. 16, pp. 1499-1506, 2009.

[20] A. P. Kuznetsov, N. V. Stankevich, and L. V. Turukina, "Coupled van der Pol-Duffing oscillators: phase dynamics and structure of synchronization tongues," Physica D: Nonlinear Phenomena, vol. 238, no. 14, pp. 1203-1215, 2009. 
[21] J.-W. Ryu, D.-S. Lee, Y.-J. Park, and C.-M. Kim, "Oscillation quenching in coupled different oscillators," Journal of the Korean Physical Society, vol. 55, no. 2, pp. 395-399, 2009.

[22] C. A. K. Kwuimy and P. Woafo, "Dynamics, chaos and synchronization of self-sustained electromechanical systems with clamped-free flexible arm," Nonlinear Dynamics, vol. 53, no. 3, pp. 201-213, 2008.

[23] J. Guckenheimer and P. Holmes, Nonlinear Oscillations, Dynamical Systems, and Bifurcations of Vector Field, Springer, New York, NY, USA, 1983.

[24] A. Wolf, J. B. Swift, H. L. Swinney, and J. A. Vastano, "Determining Lyapunov exponents from a time series," Physica D: Nonlinear Phenomena, vol. 16, no. 3, pp. 285-317, 1985.

[25] C. A. Kitio Kwuimy and P. Woafo, "Experimental realization and simulation of a self-sustained macroelectromechanical system," Mechanics Research Communications, vol. 37, no. 1, pp. 106-110, 2010.

[26] J. C. Chedjou, H. B. Fotsin, P. Woafo, and S. Domngang, "Analog simulation of the dynamics of a van der pol oscillator coupled to a duffing oscillator," IEEE Transactions on Circuits and Systems I: Fundamental Theory and Applications, vol. 48, no. 6, pp. 748757, 2001.

[27] J. Kengne, J. C. Chedjou, G. Kenne, K. Kyamakya, and G. H. Kom, "Analog circuit implementation and synchronization of a system consisting of a van der Pol oscillator linearly coupled to a Duffing oscillator," Nonlinear Dynamics, vol. 70, no. 3, pp. 2163-2173, 2012. 

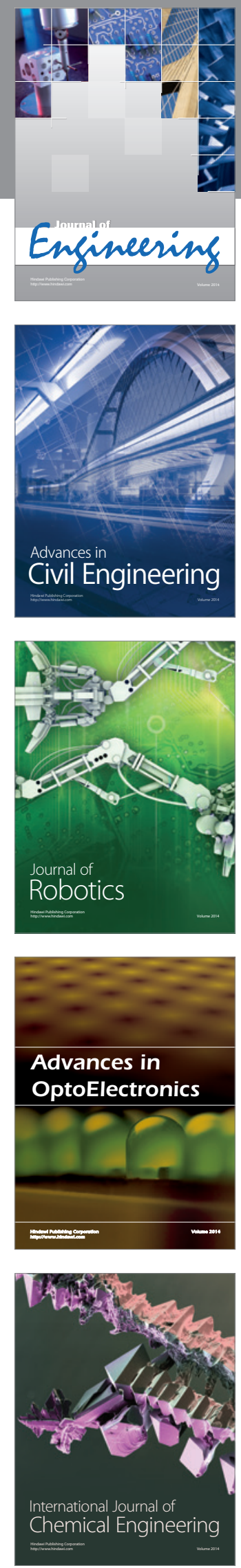

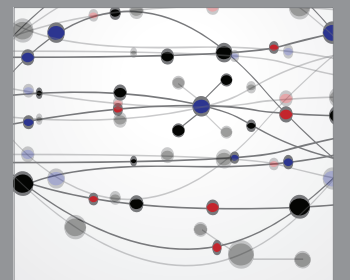

The Scientific World Journal
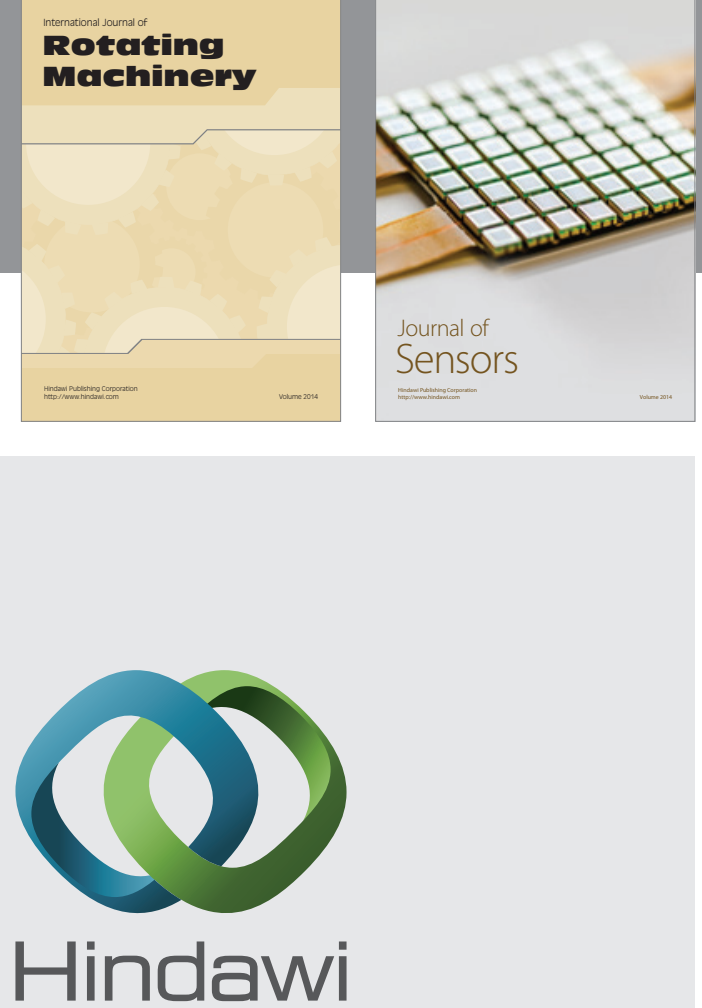

Submit your manuscripts at http://www.hindawi.com
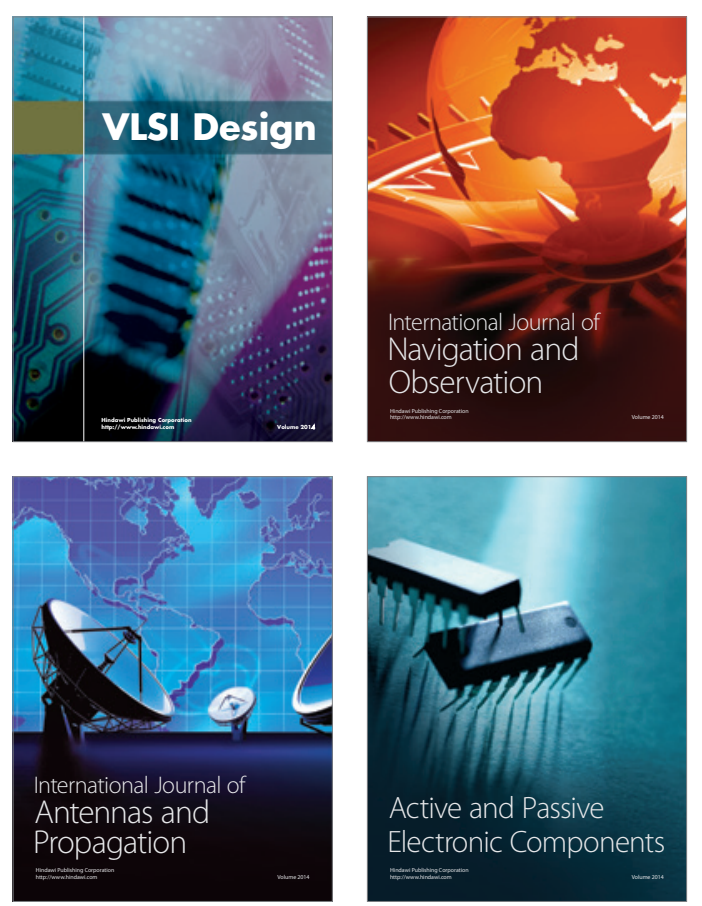
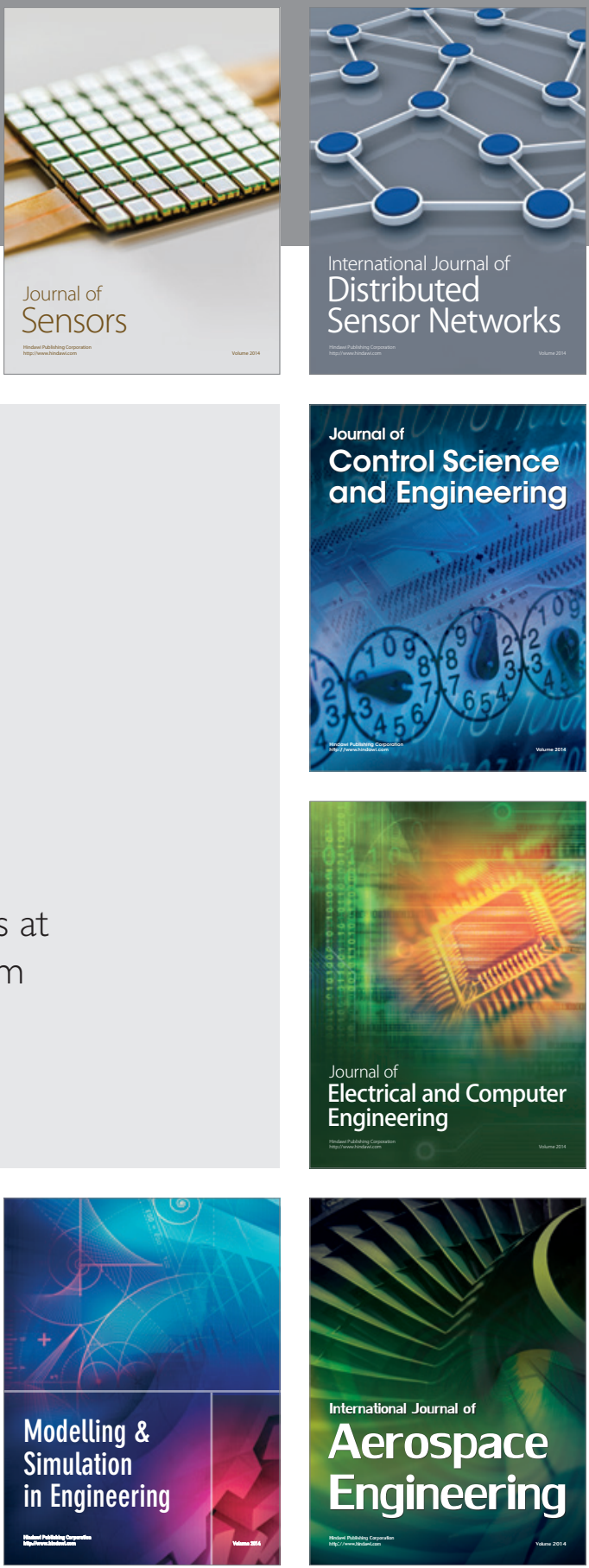

Journal of

Control Science

and Engineering
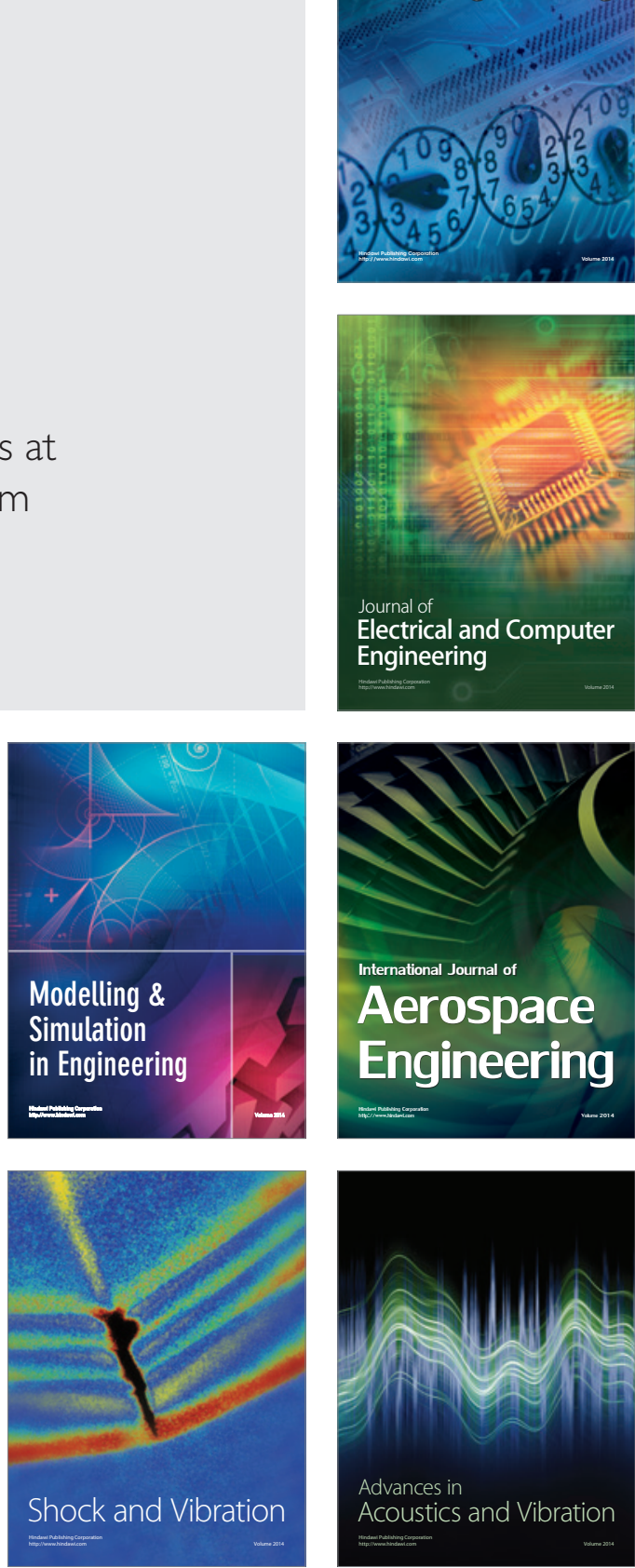